\section{Development of Normative Data using the Mini-BESTest on Children Ages 7-17}

\section{Veronica Southard ${ }^{*}$, Jennifer Hajek, Rebecca Strausman and Julie Williams}

Department of Physical Therapy, New York Institute of Technology, New York, USA

\begin{abstract}
Background: Balance deficits are often overlooked, or missed all together, in children. Catching these deficits early could potentially reduce the number of injuries these individuals may incur, without intervention. The miniBEST test is a well-regarded, psychometrically robust balance assessment, used to establish the origins of impairments in adults.
\end{abstract}

Purpose: The purpose of this study was to determine normative values for balance using the Mini-BESTest in typically developing children aged 7-17.

Methods: One hundred and one typically developing public school participants between the ages of 7-17 participated in this normative cross-sectional study. Age, gender, anthropometric measures, and activity levels were documented. Participants were assigned to groups by age; 7-10 years, 11-13, and 14-17.

Results: A significant difference was found between groups for total mean score from group one, (the youngest), when compared to groups two and three. The means based on group age are 23.84, 24.97 , and 25.43 respectively; mean scores are similar to adult normative values. The standard error of the mean (SE) assessed for these scores was .177 with a SD of $1.7895 \% \mathrm{Cl}=.17-.52$. The standard error of measurement (SEm) was calculated at 1.42 points, $95 \% \mathrm{Cl}=22.22-27.78$ points. There were no significant differences between total scores for subjects of normal weight vs. overweight/ obese. Gender was also not a significant score factor. A miniBEST item analysis found that $83.92 \%$ of subjects were unable to achieve a full score on item 14 the TUG with cognitive dual task.

Conclusion: The Mini-BESTest is an appropriate balance assessment tool for typically developing children aged 7-17.

*Corresponding author: Veronica Southard, Department of Physical Therapy, New York Institute of Technology, New York, USA. Tel: +1 5166867671. E-mail: vsouthar@nyit.edu

Citation: Southard V, Hajek J, Strausman R, Williams J (2020) Development of Normative Data using the Mini-BESTest on Children Ages 7-17. J Phys Med Rehabil Disabil 6: 49.

Received: June 22, 2020; Accepted: July 06, 2020; Published: July 20, 2020

Copyright: (C) 2020 Southard V, et al. This is an open-access article distributed under the terms of the Creative Commons Attribution License, which permits unrestricted use, distribution, and reproduction in any medium, provided the original author and source are credited.
Keywords: Balance; Children; Mini-BESTest; Normative values

\section{Introduction}

Balance is a performance-related component of physical fitness that involves the maintenance of the body's equilibrium while stationary or moving [1]. In 2016, 5.3\% of the population of children experienced dizziness or balance problems in the United States [2] of that percentage, $18.6 \%$ reported the balance problems to be a "moderate", "big", or "very big" problem [2]. Balance deficits are often overlooked, or missed all together, in children. Catching these deficits early could potentially reduce the number of injuries these individuals may incur, without intervention. This is an initiative of Healthy People 2020, to identify and treat those with balance deficits [2]. Assessing pediatric balance during yearly physicals in children with subjective complaints or in those with objective measures such as lower extremity weakness may help identify these children with balance deficits. A valid and comprehensive measure is important in ensuring development and functional independence of children [1] Obtaining data for age groups of typically developing children to reference over time is desirable.

Balance impairments have been clearly noted in numerous childhood diseases and disorders that include, but are not limited to, Cerebral Palsy (CP) [3,4] Traumatic Brain Injury (TBI) [4], Autism Spectrum Disorder (ASD) [5] and Muscular Dystrophy (MD) [6]. However, the balance measures that are commonly used for the pediatric population do not comprehensively assess standing postural control and omit some key components of mobility and independence. Sibley et al. assessed validated measures of standing balance in the pediatric population and determined the components of postural control captured in each [7]. The items included commonly described balance components of functional stability limits, underlying motor systems, static stability, verticality, reactive postural control, anticipatory postural control, dynamic stability, sensory integration and cognitive influences [7] to date, no validated pediatric measures examine all nine components of balance. In fact, most measures evaluated three or less balance components. Cognitive contributions, functional stability limits and verticality were not included in most of the tools studied. More importantly, not a single measure included reactive postural control, which is essential in children who are active.

Although there is no comprehensive test currently being used in all pediatric settings that include the majority of balance components, there is a test currently in use for adult patients that captures eight out of nine of these elements. The Mini-BESTest (a shortened version of the Balance Evaluation System Test) encompasses eight components of balance, only missing functional stability limits. This is more comprehensive than any of the current validated balance assessments currently being utilized for pediatric patients [7]. Although this test is not commonly used in pediatrics, its use in children has been tested and published in the literature. Dewar et al., examined the reproducibility of the Mini-BESTest for typically developing children and the results 
Citation: Southard V, Hajek J, Strausman R, Williams J (2020) Development of Normative Data using the Mini-BESTest on Children Ages 7-17. J Phys Med Rehabil Disabil 6: 49.

found that it can discriminate postural control abilities in children with typical development and can detect progress between days [8]. In addition, Dewar et al., found the Mini-BESTest had high inter- and intra-rater reliability using Intraclass Correlation Coefficients (ICCs $=$ .56-.86) [8]. The Mini-BESTest addresses the existing gaps in pediatric measures and it is clear that further research is recommended for this population.

The Mini-BESTest was developed using factor analysis to identify the items of the BESTest that represented dynamic balance. Rasch analysis was then used to improve the BESTest categories and eliminate some items, which amounted to 3 of the 7 domains [9]. The result was the Mini-BESTest with 14 items requires only 10-15 minutes in comparison to the BESTest which can take up to 60 minutes to administer [10]. The Mini-BESTest encompasses 4 postural control domains, as follows; anticipatory, reactive postural control, sensory orientation, and dynamic gait. The Mini-BESTest covers a broad spectrum of balance performance tasks, including dynamic body stability, transfers, gait, variation of support surfaces and of visual conditions, obstacle negotiation, reactions to external forces and performance during dual-tasking. Items are scored 0 (unable to perform or requiring help) to 2 (normal performance). The Mini-BESTest can be accessed at: http://www.bestest.us/ files/7413/6380/7277/MiniBEST_revised_final_3_8_13.pdf

Yingyongyudha et al. compared four balance evaluations including the Berg Balance Scale (BBS), Timed Up and Go (TUG), BESTest and Mini-BESTest. Results favored the Mini-BESTest over the BESTest because it demonstrated the highest ability to correctly identify adults with a history of falls, due to the Mini-BESTest's high positive and negative likelihood ratios. The Mini-BESTest has a cutoff score for identifying history of falls at 16 out of 28 , at a sensitivity of $85 \%$ and a specificity of $75 \%$. In addition, the Mini-BESTest had the highest post-test accuracy when using the suggested cutoff scores [11]. In addition, Yingyongyudha et al., found that there was no ceiling effect or responsive ceiling effect when using the Mini-BESTest unlike other balance tests such as the BBS [11]. Franchignoni et al. supported these findings, reporting that the Mini-BESTest has high validity, intrarater reliability, and interrater reliability. Additionally, this study showed that the Mini-BESTest is an accurate measure of postural balance control [9]. Although the BESTest has more content, the Mini-BESTest may be the best balance assessment tool to utilize when analyzing balance in the pediatric population because of the reduced administration time and higher accuracy [11]. This is an important consideration when considering the negligible benefit of the ICC of the BES Ttest over the miniBest [8].

The Mini-BESTest addresses gaps in pediatric measures and research, and in addition, has shown it can discriminate postural control abilities in children [8]. The lack of normative data affects the utility of this test overall as it affects the ability to recognize balance deficits, fall risks, and progress monitoring. Clinicians need to be able to utilize the information they obtain from this test to further their clinical decision making regarding their plan of care. Normative scores on the Mini-BESTest for individuals between the age of 7 to 17, would allow for its widespread use amongst pediatric patients with postural control issues, regardless of their diagnosis. It would also allow both clinicians and patients to understand and observe the deficits they currently possess as well as their progress throughout their treatment. Therefore, the purpose of this study was to develop normative data using the Mini-BESTest in typically developing children aged 7 to 17.

\section{Methods}

This one-time normative cross-sectional study was approved by the New York Institute of Technology's Institutional Review Board. Informed consent from the legal guardian and assent from each participant, age 11 and older, representing groups two and three was obtained prior to testing (Office for the Protection of Research Subjects, 2014). This was a sample of convenience.

One hundred and one children aged 7 to 17 years who participated in regular gym classes, participated in the study. Subjects were recruited from the local community, camps, and schools in the surrounding area. Our study sites also consisted of community soccer leagues for children from 4-17 years. We also collected data at a high school volleyball game. Players and their eligible siblings and friends were invited to participate. Participants were consented/assented and tested on the same day. None of the consented participants dropped out. Inclusion criteria included: children aged 7 to 17, considered to be in good health, with no medical diagnosis. Exclusion criteria included: any major musculoskeletal injury not medically cleared, any neurological, orthopedic or developmental condition, the use of any assistive devices, or any other health issues that would interfere with the subjects' safety during this study. All subjects were screened prior to testing for exclusion criteria. Demographic data for each subject was collected including height, weight, gender and shown in table 1. All subjects performed the 14 tasks of the Mini-BESTest in the same order, with step-by-step verbal instructions and demonstration. To assure uniformity, each test item instruction was read to the subject for the entire test.

All examiners were trained online, scoring methodology and administration of the Mini-BESTest. The researcher's backgrounds were as physical therapy students one year from graduation and a seasoned PT with 30 + years' experience. Reliability training was provided to all examiners. Three Mini-BESTest videos were viewed and scored by the researchers. Video was viewed by all researchers independently and scored over two consecutive days to ensure intrarater reliability. The scores were compared amongst researchers to ensure inter-rater reliability. Excellent interrater agreement was obtained .89. This reliability is in keeping with Dewar and colleagues' findings. [12].

Sample size was based on a study done by Lusardi and colleagues in the development of functional performance norms in 76 persons from 66 to 101years [13]. In this study, norms for multiple performance tests were assessed including the Berg Balance Scale and Timed Up and Go. Subjects were divided by decade, with the largest group having 34 participants. Statistics including means standard deviations, and $95 \%$ confidence intervals were calculated by decade of age, gender, and assistive device. This study was selected because, like Lusardi, this study aimed to determine the score of a typically developing children on the Mini-BESTest based on age [13]. Likewise comparing the MiniBEST score achieved of a child to an age referenced mean score and standard deviation may be useful for clinicians in determining progress, and plan of care.

\section{Data Analysis}

Statistical analyses were performed using IBM SPSS version 25. The alpha level was set at $\mathrm{p}<0.05$ for all statistical comparisons. Descriptive statistics and measures of central tendencies were run for the participants' demographic data. Analysis of variances were employed 
Citation: Southard V, Hajek J, Strausman R, Williams J (2020) Development of Normative Data using the Mini-BESTest on Children Ages 7-17. J Phys Med Rehabil Disabil 6: 49.

- Page 3 of 6 -

to the total score achieved and subscores for each balance attribute for the Mini-BESTest. Post hoc calculations were applied utilizing t-tests adjusted with the Bonferroni correction factor. Standard error of measurement and standard error of the mean were calculated. Score frequencies were assessed for each item on the miniBEST.

\section{Results}

Of the 101 subjects, $55(54 \%)$ were female (Table 1). Based on BMI percentiles, 75 (77\%) subjects were identified as normal weight (5th-85th percentile) and 14 (14\%) subjects were overweight or obese (85th percentile and greater) with the remainder of subjects classified as underweight (less than the 5th percentile.) When asked their favorite after school activity, 72 (74\%) subjects reported physically active pursuits.

Mini-BESTest total score by group means are shown in table 2 . Table 2 demonstrates significant between group differences, table 3 demonstrates where the statistically significant differences by groups (in age) were noted using ANCOVA between the groups using BMI and gender as covariates. Additionally, ANOVA results indicated there were no within group differences for the combined or subscore sections. There were no differences noted between the groups based on gender ( $\mathrm{p} .=.198)$ or BMI ( $\mathrm{p} .=.314$.)

The standard error of measurement (SEm) was calculated to be 1.42 points, $95 \% \mathrm{CI}=22.02$ to 27.78 points. This indicates that the measurement scores within this range are $95 \%$ accurate.
Score frequency was calculated for all items and is shown in figure 1. Question 14 in the dynamic gait subcategory, assessed the Timed Up and Go test with dual tasking with a cognitive component. Only $16.8 \%$ of the participants achieved a perfect score, the lowest percentage of all questions. The only other item that less than $55 \%$ of the participants scored a perfect 2 was item 5 , the backwards-reactive balance perturbation.

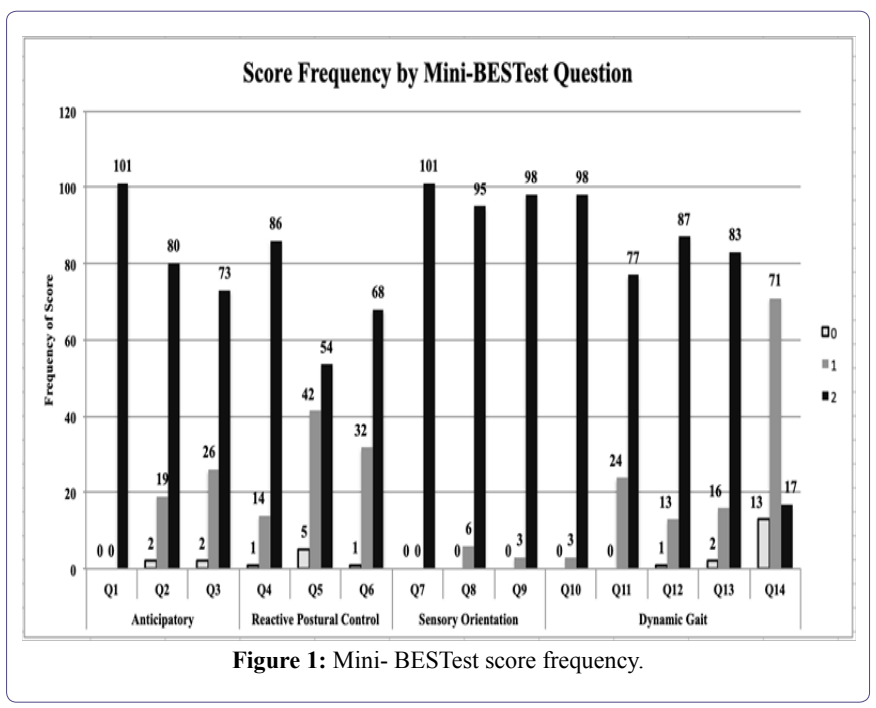

\begin{tabular}{|c|c|c|c|c|c|c|c|c|c|}
\hline \multirow{2}{*}{ Group } & \multirow{2}{*}{ Number (n) } & \multirow{2}{*}{ Average Age } & \multicolumn{2}{|c|}{ Gender } & \multirow{2}{*}{ BMI $\left(\mathbf{k g} / \mathbf{m}^{2}\right)$} & \multicolumn{4}{|c|}{ Mini-BES Test Scores } \\
\hline & & & Boys & Girls & & Mean & SD & Min & $\operatorname{Max}$ \\
\hline Group 1 (Ages 7-10) & 29 & $8.59 \pm 1.18$ & 16 & 13 & $16.04 \pm 4.45$ & 23.8 & 1.7 & 20 & 27 \\
\hline Group 2 (Ages 11-13) & 35 & $11.74 \pm 0.78$ & 12 & 23 & $18.04 \pm 5.05$ & 25.0 & 1.6 & 21 & 27 \\
\hline Group 3 (Ages 14-17) & 37 & $15.11 \pm 1.26$ & 18 & 19 & $21.58 \pm 2.52$ & 25.4 & 1.7 & 21 & 28 \\
\hline Total & 101 & $12.07 \pm 2.85$ & 46 & 55 & $18.82 \pm 4.65$ & 24.8 & 1.8 & 20 & 28 \\
\hline
\end{tabular}

Table 1: Group demographics and MiniBEST scores.

\begin{tabular}{|c|c|c|c|c|c|c|c|c|}
\hline Source & Type III Sum of Squares & df & Mean Square & F & Sig. & Partial Eta Squared & Noncent. Parameter & Observed Power \\
\hline Gender & 4.696 & 1 & 4.696 & 1.678 & .198 & .017 & 1.678 \\
\hline BMI & 3.000 & 1 & 3.000 & 1.026 & .314 & .011 & .250 \\
\hline Group & 40.820 & 2 & 20.410 & 7.292 & $.001 *$ & .131 & .171 \\
\hline
\end{tabular}

Table 2: MiniBEST Tests of Between-Subjects Effects.

b. Computed using alpha $=.05$

* Significant at .05

\begin{tabular}{|c|c|c|c|c|c|c|}
\hline \multirow{2}{*}{ Group } & \multirow{2}{*}{ Group } & \multirow{2}{*}{ Mean Difference } & \multirow{2}{*}{ Std. Error } & \multirow{2}{*}{ Sig. } & \multicolumn{2}{|c|}{$95 \%$ Confidence Interval } \\
\cline { 4 - 7 } & & & & Lower Bound & Upper Bound \\
\hline \multirow{2}{*}{1.00} & 2.00 & $-1.1438^{*}$ & .42155 & $.024^{*}$ & -2.1706 & -.1170 \\
\cline { 2 - 7 } & 3.00 & $-1.6048^{*}$ & .41635 & $.001^{*}$ & -2.6190 & -.5907 \\
\hline \multirow{2}{*}{2.00} & 1.00 & $1.1438^{*}$ & .42155 & $.024^{*}$ & .1170 & 2.1706 \\
\cline { 2 - 7 } & 3.00 & -.4610 & .39584 & .741 & -1.4252 & .5032 \\
\hline \multirow{2}{*}{3.00} & 1.00 & $1.6048^{*}$ & .41635 & $.001^{*}$ & .5907 & 2.6190 \\
\cline { 2 - 7 } & 2.00 & .4610 & .39584 & .741 & -.5032 & 1.4252 \\
\hline
\end{tabular}

Table 3: Between group differences. 
Citation: Southard V, Hajek J, Strausman R, Williams J (2020) Development of Normative Data using the Mini-BESTest on Children Ages 7-17. J Phys Med Rehabil Disabil 6: 49.

\section{Discussion}

This is the first study to assess reference data for children aged 7-17 using the Mini-BESTest. Although normative balance values have been established in older adults using an assortment of balance functional tests and measures, there is a gap in the literature in children aged 7-17, [7,13]. Previous studies have had limited sample sizes and utilize numerous functional measures instead of one tool to encompass all aspects of balance.

Our results did show a gradual increase in the scores as group members aged as well as a significant difference between the youngest age group compared to the older two groups. The means for each group were as follows: 7-10 year olds scored 23.83, 11-13 year olds scored 24.97 and 14-17 year olds scored 25.43. In comparison, the mean scores for adults aged 50-59 was 26.3 and ages $60-69$ was 24 [10]. O'Hoski et al., found a significant difference in Mini-BESTest scores between age groups in older adults between 50-89. Our review identified a gap in the literature for persons aged 18-50 of Mini-BEST scores. We did not note any ceiling effects. Other studies have also indicated the lack of ceiling effect in adults with varying conditions [9]. Post hoc Bonferroni indicated the youngest group mean scores were different from the two older groups. This can be seen in Table 2. These findings support the use of the Mini-BESTest in pediatric populations ages 7-17. Previously used measures for this age group, such as the Pediatric Balance Scale (PBS) and the Timed Up and Go (TUG), do not appear to be as effective. Franjoine et al assessed 641 subjects ages 2.3 to 13.6 , utilizing the PBS, and found that most children age 7 years and older demonstrated mastery of PBS items making it ineffective after that age [9]. Secondly, the TUG emphasizes dynamic balance in walking and movement transitions but does not examine static standing balance, which prevents it from being a fully comprehensive assessment of balance [14]. As stated in the introduction, the Mini-BESTest encompasses the majority of balance components and can discriminate amongst balance items. Therefore, the Mini-BESTest may be a more appropriate tool for identifying balance impairments, and these scores may be used as a foundation for assessing their balance ability. Additionally, due to the short administration time, this measure would serve as an excellent test for children to determine the necessity of whether to complete a lengthy protocol. Balance has been documented to be potentially compromised by anthropometric measures throughout the literature $[15,16]$. In this study, subjects classified as obese or overweight scored similarly on the Mini-BESTest in comparison with normal weight peers. A mean difference of 0.16 , indicating nonsignificant statistical difference. This study found that there are no gender differences affecting the miniBEST score.

A change in score of $2+$ points can be considered a true change not chance. Dewar and colleagues used the standard error of the mean (SE) to detect the smallest detectable change (SDC) and found that 1.3 points are required to have true change [8]. Our findings agree regarding the SDC when using the SEm. In adults, Godi et al, found the SEm to be 1.26 points, our results indicate that children are similar to adults in this regard as well [17]. Although our findings support the Mini-BESTest as an appropriate tool for determining balance deficits in children, during the TUG with dual task item (TUG COG) participants demonstrated less success than other items. As can be seen in Figure 1. This finding is consistent with other studies published in the literature. Franchignoni et al., found that participants were most likely to achieve a 1 (out of 2 possible points) on item $14[9,10]$ who conducted the Mini-BESTest on healthy adults ages 50 and up, found this section had the lowest scores and all age groups struggled with counting backwards before the physical task was added [10]. These lower scores could be attributed to a decrease in performance in situations of dual tasking, with one or both of the tasks being slightly compromised [18]. Ruffieux et al., discusses how dual tasks require more central processing and much more of a "cost" when maintaining postural control to attend to both tasks. This was noted in our study, as the children struggled to attend to both tasks. We noted that the children had significant increases in the amount of time necessary to complete the TUG COG. Hagmann-von Arx discusses the bottleneck theory and the capacity sharing theory stating that dual tasking requires cognition that is processed sequentially and when switching from one task to another, it may lead to lowered performance in one or both tasks [19]. The meta-analysis by Ruffieux recommends more research to determine the dual task effects on children, since there is not much evidence to support these theories in the articles [18]. Our study found that many children had difficulty with counting backwards whilst performing the TUG, but not necessarily due to a deficit. The subjects were able to successfully perform the TUG without the cognitive aspect, indicating lower scores could be a result of a lack of cognitive ability to perform the task rather than a balance deficit. It is not recommended to remove item 14 since dual tasking is a part of everyday life and an element of dynamic balance. Padgett et al., who found the dual-task TUG to be the least representative item of balance in the entire test when tested on 20 adults, support this observation [20-24]. Suggestions for further studies include assessing a version of the mini-BESTest that applies modifications to item 14 still requiring the use of executive functioning, but with a more age appropriate task. Dewar et al., created a version of the Mini-BESTest that is more appropriate for children called the KidsBESTest. This modifies item 14 to counting by 2's or listing random numbers [8]. This alternative should be tested in typically developing children. Item 5 , reactive balance in a posterior direction was also more difficult for this cohort of children. This was previously found by Dewar et al., whose participants had increased variability during this task [8].

A recommendation for further studies would include the development of validity of the mini-BESTest in the pediatric population as reliability and reproducibility has been established. Future studies that assess persons between the ages of 18 and 50 are warranted, to address the void in the literature and assess the validity for use with persons in this age group that have balance deficits. Another study to assess typically developing children over time using the mini BEST would be beneficial. Subjects were recruited as a sample of convenience, which can present potential limitations such as the generalizability of our results to all typically developing children aged 7-17. Our sample sizes were small. This may have created a potential limitation during our study.

The present study makes two important contributions to the existing literature by, (1) providing reference values for the MiniBESTest for children 7-17 and (2) suggesting that the Mini-BESTest could be an appropriate measure to identify change in balance deficits in the domains of the tool in children. Lastly, this study his study adds to the pool of few studies with typically developing children. 
Citation: Southard V, Hajek J, Strausman R, Williams J (2020) Development of Normative Data using the Mini-BESTest on Children Ages 7-17. J Phys Med Rehabil Disabil 6: 49.

\section{Conclusion}

The assessment of balance in the pediatric setting is essential when performing an evaluation and monitoring progress. When looking for a balance test to administer to children, therapists require a test that assesses both static and dynamic balance, can be performed in a timely manner, and can be understood by the child. It was found that the Mini-BESTest was shown to be a good tool to assess balance in typically developing children ages 7-17.

\section{References}

1. Franjoine MR, Darr N, Held SL, Kott K, Young BL (2010) The performance of children developing typically on the pediatric balance scale. Pediatric Physical Therapy: The Official Publication of the Section on Pediatrics of the American Physical Therapy Association 22: 350-359.

2. Li CM, Hoffman HJ, Ward BK, Cohen HS, Rine RM (2016) Epidemiology of dizziness and balance problems in children in the United States: A population-based study. The Journal of Pediatrics 171: 240-247.

3. Rose J, Wolff DR, Jones VK, Bloch DA, Oehlert JW, et al, (2002). Postural balance in children with cerebral palsy. Developmental Medicine and Child Neurology 44: 58-63.

4. Katz-Leurer M, Rotem H, Keren O, Meyer S (2009) Balance abilities and gait characteristics in post-traumatic brain injury, cerebral palsy and typically developed children. Developmental Neurorehabilitation 12: 100105.

5. Fournier KA, Kimberg CI, Radonovich K, Tillman MD, Chow JW, et al, (2010) Decreased static and dynamic postural control in children with autism spectrum disorders. Gait \& Posture 32: 6-9.

6. Alkan H, Mutlu A, Firat T, Bulut N, Karaduman AA, et al, (2017) Effects of functional level on balance in children with duchenne muscular dystrophy. European Journal of Pediatric Neurology: EJPN : Official Journal of the European Paediatric Neurology Society 21: 635-638.

7. Sibley KM, Beauchamp MK, Van Ooteghem K, Paterson M, Wittmeier $\mathrm{KD}$ (2017) Components of standing postural control evaluated in pediatric balance measures: A scoping review. Archives of Physical Medicine and Rehabilitation 98: 2066-2078.e4.

8. Dewar R, Claus AP, Tucker K, Ware R, Johnston LM (2017) Reproducibility of the balance evaluation systems test (BESTest) and the mini-BESTest in school-aged children. Gait \& Posture 55: 68-74.

9. Franchignoni F, Godi M, Guglielmetti S, Nardone A, Giordano A (2015) Enhancing the usefulness of the mini-BESTest for measuring dynamic balance: A rasch validation study. European Journal of Physical and Rehabilitation Medicine 51: 429-437.

10. O'Hoski S, Winship B, Herridge L, Agha T, Brooks D, et al, (2014) Increasing the clinical utility of the BESTest, mini-BESTest, and brief-BESTest: Normative values in Canadian adults who are healthy and aged 50 years or older. Physical Therapy 94: 334-342.

11. Yingyongyudha A, Saengsirisuwan V, Panichaporn W, Boonsinsukh R (2016) The mini-balance evaluation systems test (mini-BESTest) demonstrates higher accuracy in identifying older adult participants with history of falls than do the BESTest, berg balance scale, or timed up and go test. Journal of Geriatric Physical Therapy (2001) 39: 64-70.
12. Dewar R, Claus AP, Tucker K, Ware RS, Johnston LM (2019) Reproducibility of the Kids-BESTest and the Kids-Mini-BESTest for Children With Cerebral Palsy. Archives of Physical Medicine and Rehabilitation 100: 695-702.

13. Lusardi MM, Pellecchia GL, Schulman M (2003) Functional performance in community living older adults. Journal of Geriatric Physical Therapy (2001) 26: 14-22.

14. Williams EN, Carroll SG, Reddihough DS, Phillips BA, Galea MP (2005) Investigation of the timed up \& go) test in children. Developmental Medicine and Child Neurology 47: 518-524.

15. Lara S, Graup S, Balk RS, Teixeira LP, Farias AD, et al, (2017) Association between postural balance and anthropometric indexes in elementary school children. [Associacao Entre O Equilibrio Postural E Indicadores Antropometricos Em Escolares] Revista Paulista De Pediatria : Orgao Oficial Da Sociedade De Pediatria De Sao Paulo 36: 00011.

16. Nunez-Gaunaurd A, Moore JG, Roach KE, Miller TL, Kirk-Sanchez NJ (2013) Motor proficiency, strength, endurance, and physical activity among middle school children who are healthy, overweight, and obese. Pediatric Physical Therapy: The Official Publication of the Section on Pediatrics of the American Physical Therapy Association 25: 130-138

17. Godi M, Franchignoni F, Caligari M, Giordano A, Turcato AM, et al, (2013) Comparison of reliability, validity, and responsiveness of the mini-BESTest and Berg Balance Scale in patients with balance disorders. Phys Ther Feb 93: 158-167.

18. Ruffieux J, Mouthon A, Keller M, Mouthon M, Annoni JM, et al. (2018) Balance training reduces brain activity during motor simulation of a challenging balance task in older adults: An fMRI study. Frontiers in Behavioral Neuroscience 12: 10.

19. Hagmann-von Arx P, Manicolo O, Lemola S, Grob A (2016) Walking in school-aged children in a dual-task paradigm is related to age but not to cognition, motor behavior, injuries, or psychosocial functioning. Frontiers in Psychology 7: 352.

20. Padgett PK, Jacobs, JV, Kasser SL (2012) Is the BESTest at its best? A suggested brief version based on interrater reliability, validity, internal consistency, and theoretical construct. Physical Therapy 92: 1197-1207.

21. Alves RF, Rossi AG, Pranke GI, Lemos LF (2012) Influence of gender in postural balance of school age children. Revista CEFAC 15: 528-537.

22. Assent from Children: Office for the Protection of Research Subjects. (2014, November 13).

23. Ruiz LM, Graupera JL, Gutiérrez M, Miyahara M (2003) The assessment of motor coordination in children with the movement $\mathrm{ABC}$ test: A comparative study among Japan, USA and Spain. International Journal of Applied Sports Science 15: 22-35.

24. Schedler S, Kiss R, Muehlbauer T (2019) Age and sex differences in human balance performance from 6-18 years of age: A systematic review and meta-analysis. PloS One 14: e214434.

\section{Appendix}

http://www.bestest.us/files/7413/6380/7277/MiniBEST_revised_final_3_8_13.pdf (accessed 1/7/20) 


\section{If}

Advances In Industrial Biotechnology | ISSN: 2639-5665

Advances In Microbiology Research | ISSN: 2689-694X

Archives Of Surgery And Surgical Education | ISSN: 2689-3126

Archives Of Urology

Archives Of Zoological Studies | ISSN: 2640-7779

Current Trends Medical And Biological Engineering

International Journal Of Case Reports And Therapeutic Studies | ISSN: 2689-310X

Journal Of Addiction \& Addictive Disorders | ISSN: 2578-7276

Journal Of Agronomy \& Agricultural Science | ISSN: 2689-8292

Journal Of AIDS Clinical Research \& STDs | ISSN: 2572-7370

Journal Of Alcoholism Drug Abuse \& Substance Dependence | ISSN: 2572-9594

Journal Of Allergy Disorders \& Therapy | ISSN: 2470-749X

Journal Of Alternative Complementary \& Integrative Medicine | ISSN: 2470-7562

Journal Of Alzheimers \& Neurodegenerative Diseases | ISSN: 2572-9608

Journal Of Anesthesia \& Clinical Care | ISSN: 2378-8879

Journal Of Angiology \& Vascular Surgery | ISSN: 2572-7397

Journal Of Animal Research \& Veterinary Science | ISSN: 2639-3751

Journal Of Aquaculture \& Fisheries | ISSN: 2576-5523

Journal Of Atmospheric \& Earth Sciences | ISSN: 2689-8780

Journal Of Biotech Research \& Biochemistry

Journal Of Brain \& Neuroscience Research

Journal Of Cancer Biology \& Treatment | ISSN: 2470-7546

Journal Of Cardiology Study \& Research | ISSN: 2640-768X

Journal Of Cell Biology \& Cell Metabolism | ISSN: 2381-1943

Journal Of Clinical Dermatology \& Therapy | ISSN: 2378-8771

Journal Of Clinical Immunology \& Immunotherapy | ISSN: 2378-8844

Journal Of Clinical Studies \& Medical Case Reports | ISSN: 2378-8801

Journal Of Community Medicine \& Public Health Care | ISSN: 2381-1978

Journal Of Cytology \& Tissue Biology | ISSN: 2378-9107

Journal Of Dairy Research \& Technology | ISSN: 2688-9315

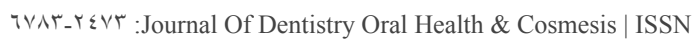

Journal Of Diabetes \& Metabolic Disorders | ISSN: 2381-201X

Journal Of Emergency Medicine Trauma \& Surgical Care | ISSN: 2378-8798

Journal Of Environmental Science Current Research | ISSN: 2643-5020

Journal Of Food Science \& Nutrition | ISSN: 2470-1076

Journal Of Forensic Legal \& Investigative Sciences | ISSN: 2473-733X

Journal Of Gastroenterology \& Hepatology Research | ISSN: 2574-2566
Journal Of Genetics \& Genomic Sciences | ISSN: 2574-2485

Journal Of Gerontology \& Geriatric Medicine | ISSN: 2381-8662

Journal Of Hematology Blood Transfusion \& Disorders | ISSN: 2572-2999

Journal Of Hospice \& Palliative Medical Care

Journal Of Human Endocrinology | ISSN: 2572-9640

Journal Of Infectious \& Non Infectious Diseases | ISSN: 2381-8654

Journal Of Internal Medicine \& Primary Healthcare | ISSN: 2574-2493

Journal Of Light \& Laser Current Trends

Journal Of Medicine Study \& Research | ISSN: 2639-5657

Journal Of Modern Chemical Sciences

Journal of Nanotechnology Nanomedicine \& Nanobiotechnology | ISSN: 2381-2044

Journal Of Neonatology \& Clinical Pediatrics | ISSN: 2378-878X

Journal Of Nephrology \& Renal Therapy | ISSN: 2473-7313

Journal Of Non Invasive Vascular Investigation | ISSN: 2572-7400

Journal Of Nuclear Medicine Radiology \& Radiation Therapy | ISSN: 2572-7419

Journal Of Obesity \& Weight Loss | ISSN: 2473-7372

Journal Of Ophthalmology \& Clinical Research | ISSN: 2378-8887

Journal Of Orthopedic Research \& Physiotherapy | ISSN: 2381-2052

Journal Of Otolaryngology Head \& Neck Surgery | ISSN: 2573-010X

Journal Of Pathology Clinical \& Medical Research

๑ รุ_หษ १: Journal Of Pharmacology Pharmaceutics \& Pharmacovigilance | ISSN

Journal Of Physical Medicine Rehabilitation \& Disabilities | ISSN: 2381-8670

Journal Of Plant Science Current Research | ISSN: 2639-3743

Journal Of Practical \& Professional Nursing | ISSN: 2639-5681

Journal Of Protein Research \& Bioinformatics

Journal Of Psychiatry Depression \& Anxiety | ISSN: 2573-0150

Journal Of Pulmonary Medicine \& Respiratory Research | ISSN: 2573-0177

Journal Of Reproductive Medicine Gynaecology \& Obstetrics | ISSN: 2574-2574

Journal Of Stem Cells Research Development \& Therapy | ISSN: 2381-2060

Journal Of Surgery Current Trends \& Innovations | ISSN: 2578-7284

Journal Of Toxicology Current Research | ISSN: 2639-3735

Journal Of Translational Science And Research

Journal Of Vaccines Research \& Vaccination | ISSN: 2573-0193

Journal Of Virology \& Antivirals

Sports Medicine And Injury Care Journal | ISSN: 2689-8829

Trends In Anatomy \& Physiology | ISSN: 2640-7752

Submit Your Manuscript: https://www.heraldopenaccess.us/submit-manuscript 